\title{
Compact Ultra-wideband Bandpass Filter with Wide Fractional Bandwidth and High Skirt Selectivity
}

\author{
Lei Bai ${ }^{\mathrm{a}}$, Yi-qi Zhuang ${ }^{\mathrm{a}}$, Zhi-bin Zeng ${ }^{\mathrm{a},{ }^{*}}$ \\ ${ }^{a}$ School of Microelectronics, Xidian University,Xi'an 710071, China
}

Received: 10 September 2019; Accepted: 15 October 2019; Published: 08 November 2019

\begin{abstract}
A compact ultra-wideband bandpass filter having highly selectivity and wide bandwidth simultaneously is proposed, which adopts a modified multiple-mode resonator (MMR) and annular structures. The modified MMR is constructed by multiple uniform-impedance stubs and a dumbbell-based stepped-impedance stub. Two transmission zeros are created by the dumbbell-based stepped-impedance stub at the lower and upper cutoff frequencies to improve the selectivity. Additionally, two annular defected ground structures in the aperture ground are adopted to enhance the coupling factor and reduce the etching size. This bandpass filter has a fractional bandwidth of $127 \%$ with selectivity of up to 0.939 . The measured results are in good agreement with the simulation results.
\end{abstract}

Index Terms: Ultra-wideband (UWB), multiple-mode resonator (MMR), dumbbell-based stepped-impedance stub, annular defected ground structures

(C) 2019 Published by MECS Publisher. Selection and/or peer review under responsibility of the Research Association of Modern Education and Computer Science

\section{Introduction}

Since the Federal Communication Commission (FCC) first authorized the unlicensed frequency band of 3.110.6 GHz for commercial ultra-wideband (UWB) system ${ }^{[1,2]}$, an ultra-wideband (UWB) bandpass filter (BPF) has become an essential component of a UWB system, which plays a significant role in UWB wireless communication. Lately, many different models have been introduced to develop new UWB bandpass filters, including composite lowpass/highpass filter topology $\mathrm{y}^{[3]}$, planar balum ${ }^{[4]}$, broadside-coupled structure ${ }^{[5]}$, defected microstrip structure ${ }^{[6]}$, cross coupling structure ${ }^{[7]}$, parasitic coupled line ${ }^{[8]}$, and multiple-mode

* Corresponding author.

E-mail address: zbzeng@163.com 
resonator (MMR) ${ }^{[9-15]}$. The above structures have some good performance, such as low insertion loss, wide bandwidth, sharp skirt selectivity and compact size. It is challenged to design multiple good performance simultaneously.

For selectivity, stub-loaded resonators are often used to achieved good skirt selectivity. Chu et al. ${ }^{[10]}$ proposed a compact UWB BPF by loading three open stubs in a uniform-impedance resonator with a fractional bandwidth of $117 \%$, and achieved selectivity of up to 0.921. Reference [16] designed a compact UWB filter using dual-stub-loaded resonators (DSLR) to realize good skirt selectivity and harmonic suppression. For compact size, MMR is one of the most suitable design method for miniaturized UWB filters. An UWB bandpass filter with fan-shaped stub-loaded MMR and wide stopband was presented in [17], the filter could exhibit wide stopband. Shi et al. ${ }^{[14]}$ proposed a modified multiple-mode resonator combined with two aperturebacked interdigital coupled-lines to obtain sharp rejection skirts. Aditi Sengupta et al. ${ }^{[18]}$ proposed a miniature sized UWB filter using a dual MMR and a DGS, the simulated resulted could reach a wide fractional bandwidth of 119\%. Furthermore, in [19], the EBG-loaded technique and the cascaded high-/low-pass filters were studied to extend the stopband. In order to improve the performance of filters based on parallel-coupled lines. $\mathrm{Wu}^{[20]}$ proposed transversal signal-interaction concepts to improve the filter performance. Xia et al. ${ }^{[21]}$ presented a compact UWB filter with asymmetric parallel-coupled lines (APCL) combine with open and short stubs, which could improve the frequency selectivity. The above methods have either relatively high selectivity or relatively high fractional bandwidth, i.e., they could not exhibit high selectivity and high fractional bandwidth simultaneously.

In this paper, we propose a compact modified multiple-mode resonator (MMR) based on symmetrical parallel-coupled feed lines (see Fig.1), which is constructed by loading multiple open stubs and a dumbbellbased stepped-impedance open-stub. The proposed UWB BPF could generate two transmission zeros in the lower and upper bands and five modes in the desired passband. On one hand, multiple stubs at the symmetrical sides could increase the transmission poles and enhance out-of-band rejection. In addition, a dumbbell-based stepped-impedance stub at the center could improve the fractional bandwidth. On the other hand, two annular aperture-backed structures in a ground plane are introduced to enhance the coupling degree of the coupled lines. This paper aims at combining sharp skirt selectivity with fractional bandwidth. Both simulation and measurement are in good agreement, showing good wideband filtering performance and high selectivity.

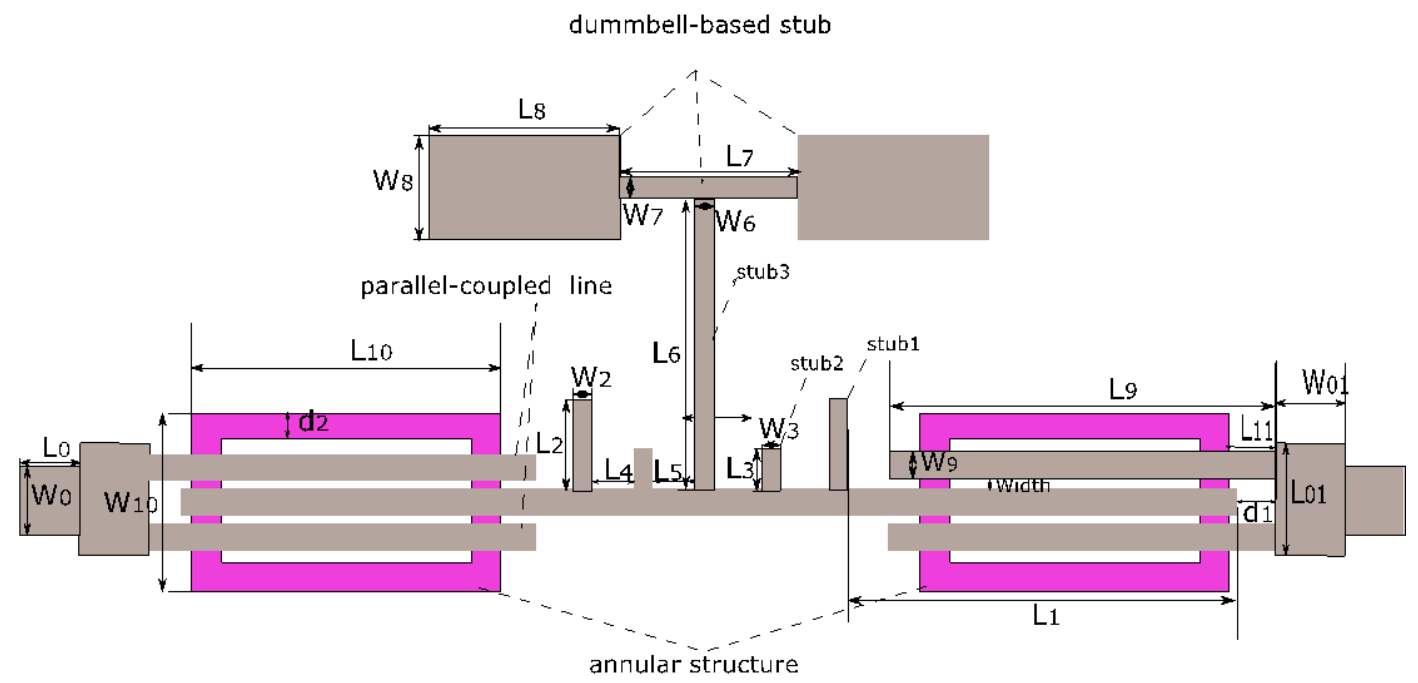

Fig.1. Schematic of the proposed UWB filter. 


\section{Basic filter design and analysis}

A UWB filter is proposed based on a modified MMR and two annular aperture-backed structures. As shown in Fig.1, the basic structure is constructed using uniform-impedance open stubs (stub1 and stub2) of width $\mathrm{W}_{2}$, length $\left(\mathrm{L}_{2}, \mathrm{~L}_{3}\right)$ at the symmetrical sides, and a dumbbell-based stepped-impedance stub (stub3) of width $\left(\mathrm{W}_{8}\right)$, length $\left(\mathrm{L}_{8}\right)$ at the center.

Because the proposed modified MMR is symmetrical in structure, it employs the odd- and even-mode method, which is widely used for analyzing symmetrical structures. Fig.2 exhibits the equivalent circuits of odd-mode and even-mode excitations.

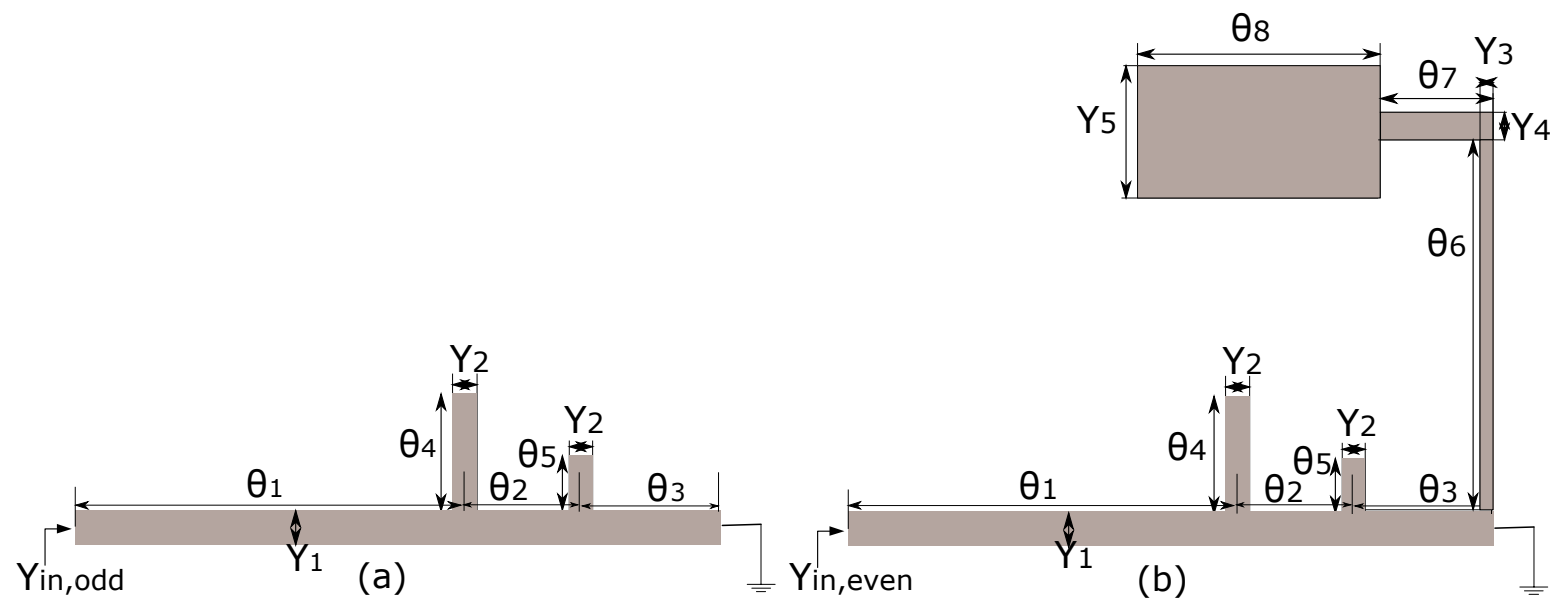

Fig. 2. (a) odd-mode equivalent circuit of the resonator. (b) even-mode equivalent of the resonator.

For the odd-mode and even-mode cases, the input admittance $\mathrm{Y}_{\mathrm{in}, \text { odd }}$ and $\mathrm{Y}_{\mathrm{in}, \mathrm{even}}$ can be derived as follows:

$$
Y_{\text {in,odd }}=Y_{1} \frac{Y_{1 o}+j Y_{1} \tan \theta_{1}}{Y_{1}+j Y_{1 o} \tan \theta_{1}}, Y_{\text {in,even }}=Y_{1} \frac{Y_{1 e}+j Y_{1} \tan \theta_{1}}{Y_{1}+j Y_{1 e} \tan \theta_{1}}
$$

Where

$$
\begin{gathered}
Y_{1 o}=j Y_{2} \tan \theta_{4}+Y_{1} \frac{j Y_{2} \tan \theta_{5}-j Y_{1} \cot \theta_{3}+j Y_{1} \tan \theta_{2}}{Y-Y_{2} \tan \theta_{5}-j Y_{1} \cot \theta_{3} \tan \theta_{2}} \\
Y_{1 e}=j Y_{2} \tan \theta_{4}+Y_{1} \frac{j Y_{2} \tan \theta_{5}-j Y_{1} \cot \theta_{3}+j Y_{1} \tan \theta_{2}}{Y-Y_{2} \tan \theta_{5}-j Y_{1} \cot \theta_{3} \tan \theta_{2}} \\
Y_{2 e}=j Y_{2} \tan \theta_{5}+Y_{1} \frac{j Y_{3 e}+j Y_{1} \tan \theta_{3}}{Y_{1}+j Y_{3 e} \tan \theta_{3}}
\end{gathered}
$$




$$
Y_{3 e}=Y_{3} \frac{Y_{4} \frac{Y_{5} \tan \theta_{8}+j Y_{4} \tan \theta_{7}}{Y_{4}-Y_{5} \tan \theta_{7} \tan \theta_{8}}+j Y_{3} \tan \theta_{6}}{Y_{1}+j Y_{4} \frac{j Y_{5} \tan \theta_{8}+j Y_{4} \tan \theta_{7}}{Y_{4}-Y_{5} \tan \theta_{7} \tan \theta_{8}} \tan \theta_{6}}
$$

From the resonance condition $\mathrm{Y}_{\mathrm{in}, \mathrm{odd}}=0$ and $\mathrm{Y}_{\mathrm{in}, \mathrm{even}}=0$, the resonate modes of odd-mode and even-mode could be extracted:

$$
Y_{1 o}+j Y_{1} \tan \theta_{1}=0, \quad Y_{1 e}+j Y_{1} \tan \theta_{1}=0
$$

$L_{2}, L_{3}, L_{8}, W_{8}$ are varied to obtain some effect on the resonant frequencies $f_{1}, f_{2}, f_{3}, f_{4}, f_{5}$ and the transmissions zeros $f_{\mathrm{z} 1}$ and $f_{\mathrm{z} 2}$. Fig. 3 (a) and Fig.3 (b) shows a simulated characteristics of the proposed modified MMR under the weak coupling case with the varied $L_{2}$ and $L_{3}$. As $L_{2}$ changes from 2.35 to $3.15 \mathrm{~mm}$, and $L_{3}$ changes from 0.4 to $2.4 \mathrm{~mm}$. We could find that it has a small effect on $f_{1}, f_{2}, f_{3}$, while $f_{4}, f_{5}$ obviously change. Meanwhile, $f_{\mathrm{z} 1}$ is almost invariable and $f_{\mathrm{z} 2}$ obviously changes. The odd-mode resonance of the proposed modified MMR depends on multiple uniform-impedance stubs.

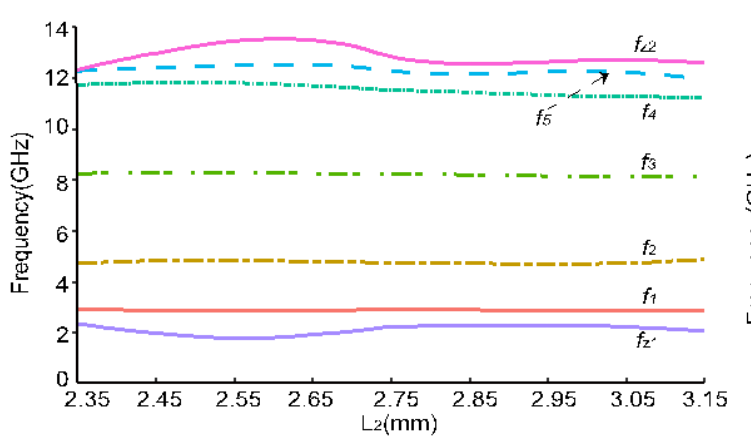

(a)

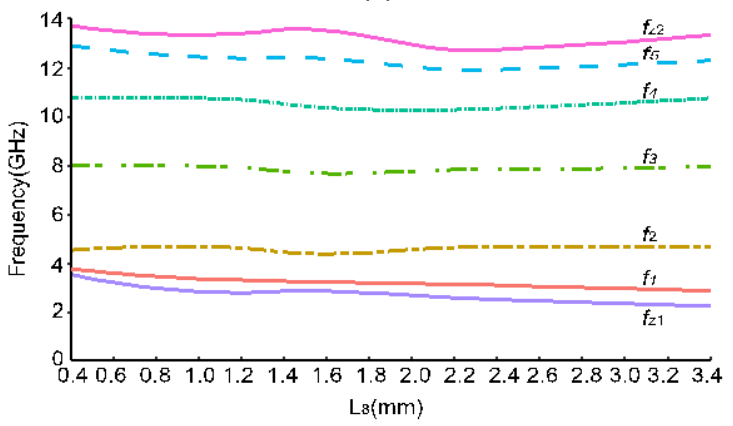

(c)

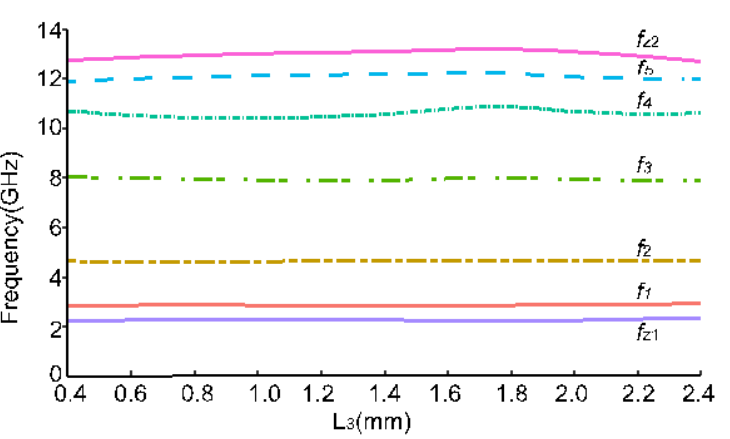

(b)

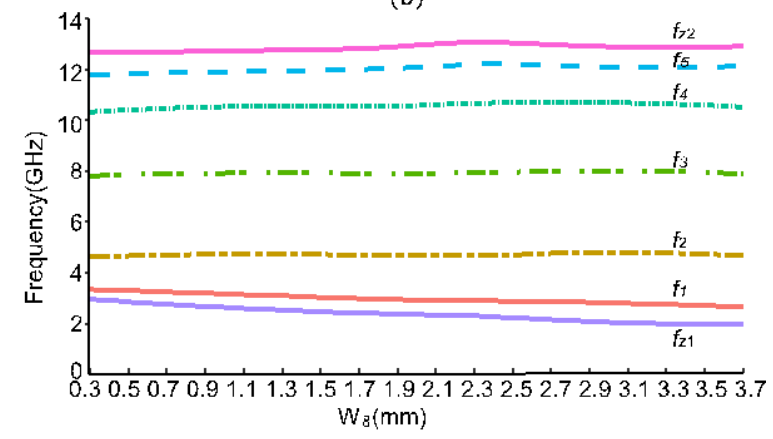

(d)

Fig. 3.Resonant frequencies under different (a) $\mathrm{L}_{2}$, (b) $\mathrm{L}_{3}$, (c) $\mathrm{L}_{8}$ and (d) $\mathrm{W}_{8}$.

It is evident from (6) that even-mode resonance conditions can be mainly controlled by adjusting structural parameters $\left(L_{8}\right.$ and $\left.W_{8}\right)$ of the resonator. The even-mode of the modified MMR is linked to the solutions of (6). It can be seen from Fig. 3 (c), as length $L_{8}$ varied from 0.4 to $3.4 \mathrm{~mm}$, the $f_{1}$ and $f_{5}$ decrease dramatically, while $f_{3}$ fixed, with little change in $f_{2}$ and $f_{4}$ being observed. As $W_{8}$ varies from 0.2 to $3.6 \mathrm{~mm}$ (see Fig.3 (d)), $f_{1}$ 
decreases dramatically while $f_{5}$ increases slowly, and $f_{2}$ fixed while $f_{3}$ and $f_{4}$ little change. Finally, the two transmission zeros $f_{\mathrm{z} 1}$ and $f_{\mathrm{z} 2}$ have the same tendency as $f_{1}$ and $f_{5}$. The even-mode of modified MMR depends on the stepped-impedance stub at the center.

In addition, in the aperture ground, it uses the annular defected ground structure instead of rectangular structure to enhance the coupling factor and reduce the etching area. The backside aperture on the ground plane is formed not only to tighten the coupling degree of coupled lines and also to realize the specified impedance ratio of side to central sections in the MMR. Fig.4 (a) is the comparison of S parameter with different structures. When there is no aperture in the aperture ground, it has a fluctuates greatly in bandpass ripple. Adding rectangular defected ground structure could improve flatness greatly. It can be seen that the annular structure have two advantages. Firstly, it can improve the in-band flatness $(8-9 \mathrm{GHz})$ and have the insertion loss less than $0.66 \mathrm{~dB}$ in passband. Secondly, it could improve the stop-band rejection. Moreover, there are five modes of resonant frequencies within passband and two transmission zeros under weak coupling (see Fig.4 (b)). The transmission zeros can be created in the desired passband to achieve good selectivity. By adopting symmetrical parallel-coupled feed lines and two annular aperture-backed structures in the proposed UWB filter, a UWB filter with a good passband performance and high skirt selectivity is realized.

\section{Simulation and Measurement Results}

In order to verify the proposed concept, a UWB bandpass filter with a modified MMR and two annular structures is fabricated and measured. It is fabricated on an RT/Duroid 5880 substrate with a relative constant of 2.2 and thickness of a $0.762 \mathrm{~mm}$. Dimensions are selected as follows: $L_{01}=2.5, L_{0}=1.5, L_{1}=8.58, L_{2}=3.3$, $L_{3}=1.93, L_{4}=0.5, L_{5}=0.82, L_{6}=9.2, L_{7}=1.7, L_{8}=3.4, L_{9}=7.71, L_{10}=6.4, L_{11}=0.61, W_{01}=1, W_{0}=1.5, W_{2}=0.5, W_{3}=0.5$, $W_{6}=0.4, W_{7}=0.6, W_{8}=2.2, W_{9}=0.4, W_{10}=2$, width $=0.1, d_{1}=0.21, d_{2}=0.72$ (all in millimeters).

A comparison between the proposed work and [10] indicates the presence of similarities in structure; for example, both studies adopt stub-loaded multiple mode resonator and a parallel coupled feeding lines. However, there are several structural differences between both studies. First, in [10], step impedance open circuit branches are used in the middle plane, whereas in this work, dumbbell step impedance open circuit branches are used, which increase the relative percentage bandwidth. Moreover, in [10], a symmetrical single open branch is adopted on both sides of the central step impedance branch. However, in this work, a symmetrical multi-open branch is adopted, which increases transmission poles and enhances out-of-band suppression. In addition, in [10], a rectangular defect ground structure is adopted, whereas in this work, an annular defected

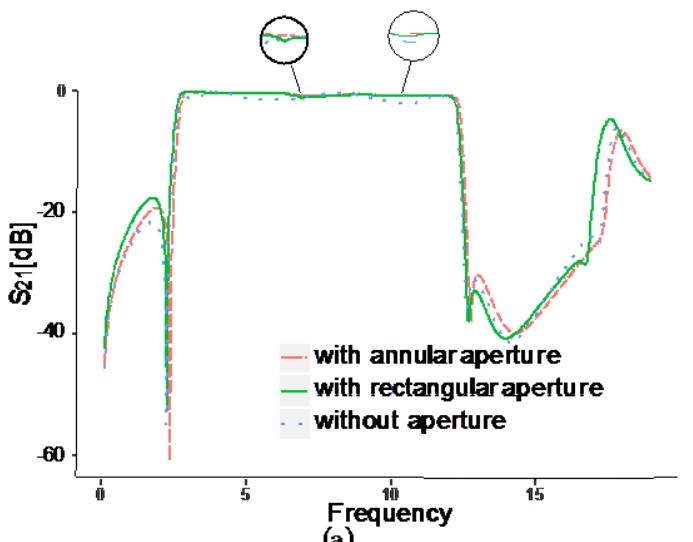

(a)

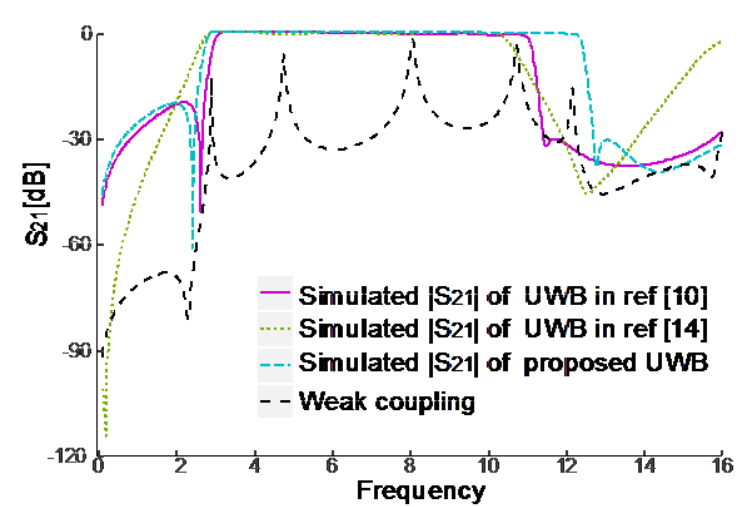

(b)

Fig. 4. (a) Comparison of simulated $\left|S_{21}\right|$ of different aperture ground. (b) Comparison of the simulated $\left|S_{21}\right|$ of the proposed UWB BPF against those shown in [10] and [14]. 
ground structure that enhances flatness and reduces the etching area is used. Fig.4 (b) depicts a comparison of the simulated $\left|S_{21}\right|$ of the proposed UWB BPF against those shown in [10] and [14]. As is shown in Fig.4 (b), there are five modes resonant frequencies, and two transmission zeros, under weak coupling. In addition, the proposed UWB filter has a better performance in fractional bandwidth than previous work.

The simulation results using ansoft HFSS 15.0 software shows a wide bandwidth and high skirt selectivity. The fabricated circuit, shown in Fig.5 (a), is tested using Agilent's Vector Network Analyzer (E8363B). Fig.5 (b) shows the simulated and measured results of the UWB bandpass filter. The $3 \mathrm{~dB}$ passband covers the range of $2.87-12.28 \mathrm{GHz}$, while the counterpart frequency of $2.76-12.37 \mathrm{GHz}$ in simulation. The skirt factor is 0.939 with a fractional bandwidth of $127 \%$. The average insertion loss is $0.9 \mathrm{~dB}$. The overall size of fabricated wideband $\mathrm{BPF}$ is $12.95 \times 27.65 \mathrm{~mm}^{2}$, which amounts to $0.63 \lambda_{0} \times 0.29 \lambda_{0}$. The measured return loss is less than $10.5 \mathrm{~dB}$. Some slight deviations were observed between the simulation and measurement due to the fabrication error.
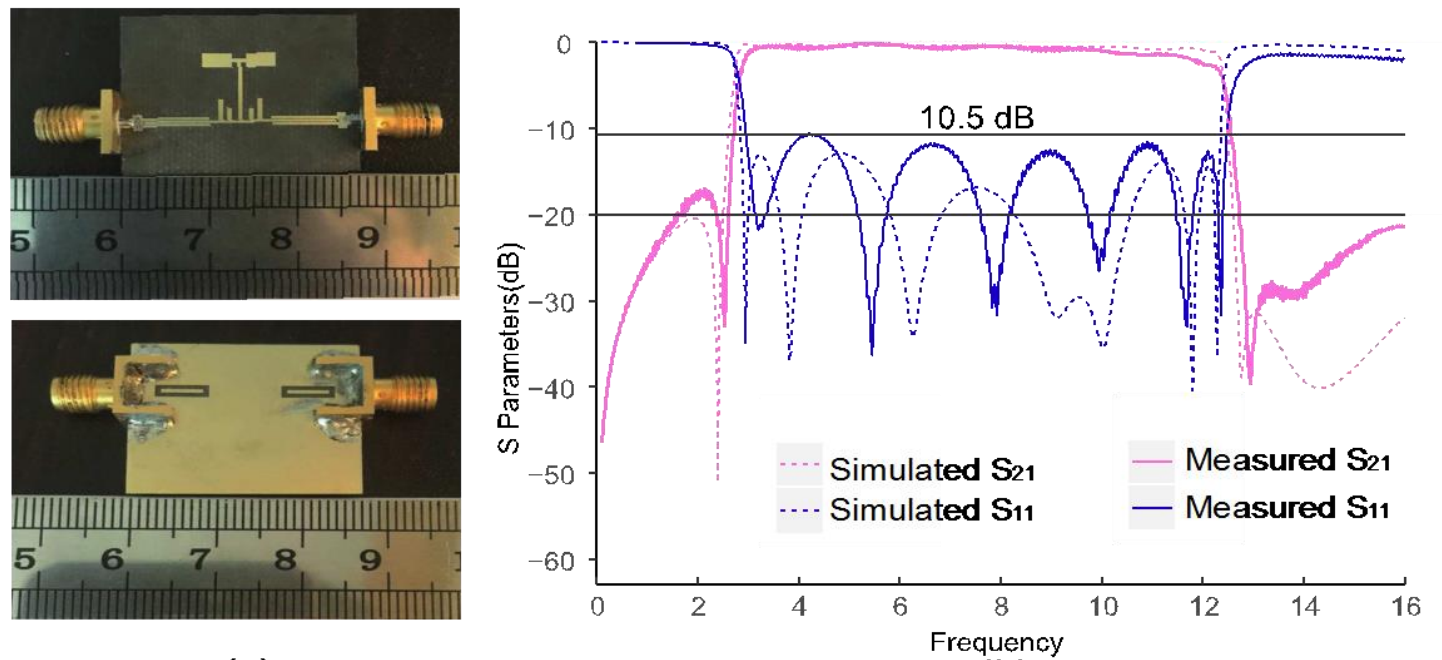

(a)

(b)

Fig.5. (a) Photograph of the fabricated UWB filter. (b) Simulated and measured results of the designed UWB filter.

Table 1 compares the proposed UWB filter with others in literature and includes the following specifications: skirt factor (S.F.) and fractional bandwidth (FBW) .

Table 1. Comparison with the reported UWB BPFs

\begin{tabular}{c|c|c|c|c}
\hline Ref & $\varepsilon_{\mathrm{r}}$ & FBW $(3 \mathrm{~dB})$ & S.F. & Size $\left(\lambda_{0} \times \lambda_{0}\right)$ \\
\hline$[7]$ & $2.45 / 0.8$ & $108 \%$ & 0.804 & $0.63 \times 0.46$ \\
\hline$[10]$ & $2.55 / 0.8$ & $117 \%$ & 0.921 & $0.514 \times 0.312$ \\
\hline$[14]$ & $2.55 / 0.8$ & $126 \%$ & 0.802 & $0.58 \times 0.12$ \\
\hline$[16]$ & $2.55 / 0.8$ & $122 \%$ & NA & $0.51 \times 0.33$ \\
\hline$[18]$ & $4.4 / 1.6$ & $119 \%$ & NA & $0.454 \times 0.295$ \\
\hline$[21]$ & $3.66 / 0.5 .8$ & $109.5 \%$ & 0.8 & $0.645 \times 0.318$ \\
\hline TW & $2.2 / 0.8$ & $127 \%$ & 0.939 & $0.628 \times 0.294$ \\
\hline
\end{tabular}

NA=not available; $\lambda_{0}$ is the free space of wavelength at $6.85 \mathrm{GHz}$;

S.F. is the skirt factor of the passband; 
From Table 1, it can be seen, the proposed UWB BPF has sharper skirt selectivity (S.F.=0.939) than those previously reported. Compared with ref [10], it increased by 0.018 in S. F. In addition, the bandwidth of UWB filter proposed in this paper is better than that of previous works. Compared with the references [16] and [18], the bandwidth of UWB filter is improved by $5 \%$ and $8 \%$, respectively. Although it has almost the same fractional bandwidth as in [14], the proposed UWB filter has better skirt selectivity, where the S.F. increased by 0.137 . Therefore, the proposed UWB filter has advantages in fractional bandwidth and skirt selectivity.

\section{Conclusions}

This letter proposed an ultra-wideband bandpass filter based on a modified multiple-mode resonator (MMR) and annular defected ground structure. The modified MMR was constructed by loading multiple uniformimpedance stubs and a dumbbell-based stepped-impedance stub. Additionally, two annular defect ground structure in the ground plane were introduced to enhance the coupling degree of the symmetrical parallelcoupled feed lines and reduce the etching size. The filter successfully demonstrated sharp skirt selectivity, low insertion loss, good passband performance, and wide bandwidth. It is suitable for application to UWB systems owing to its advantages such as wide fractional bandwidth and good skirt selectivity, but the size is relatively large. Next, we can further optimize the size. What's more, although the bandwidth and selectivity of this letter are greatly improved, There are other communication systems interference in the UWB range, such as $3.6 \mathrm{GHz}$ WiMAX, 5.8 GHz WLAN and $8.0 \mathrm{GHz}$ satellite communication systems. We can design an UWB filter with multiple notched band in the future.

\section{References}

[1] Zhu L, Menzel W. Compact microstrip bandpass filter with two transmission zeros using a stub-tapped half-wavelength resonator[J]. IEEE Microw Guided Wave Lett, 2003, 13(1): 16-18.

[2] Zhu L, Sun S, Menzel W. Ultra-Wideband (UWB) bandpass filters using multiple-mode resonator[J]. IEEE Microwave \& Wireless Components Letters, 2005, 15(11): 796-798.

[3] Ghatak R, Sarkar P, Mishra R K, et al. A Compact UWB Bandpass Filter With Embedded SIR as Band Notch Structure[J]. IEEE Microwave \& Wireless Components Letters, 2011, 21(5): 261-263.

[4] Feng W J, Chen W Q. Ultra-wideband bandpass filter using broadband planar Marchand balun[J]. Electronics Letters, 2011, 47(3): 198.

[5] Keren L I, Yamamoto Y, Kurita D, et al. An Ultra-Wideband (UWB) Bandpass Filter Using BroadsideCoupled Structure and Lumped-Capacitor-Loaded Shunt Stub Resonators[J]. Ieice Transelectronc, 2007, 90(9): 1736-1742.

[6] Fallahzadeh S, Tayarani M. A New Microstrip UWB Bandpass Filter Using Defected Microstrip Structures[J]. Journal of Electromagnetic Waves \& Applications, 2010, 24(7): 893-902.

[7] Li X, Ji X. Novel Compact UWB Bandpass Filters Design With Cross-Coupling Between lambda/4 Short-Circuited Stubs[J]. IEEE Microwave and Wireless Components Letters, 2014, 24(1): 23-25.

[8] Pirani S, Nourinia J, Ghobadi C. Band-notched UWB BPF design using parasitic coupled line[J]. IEEE Microwave \& Wireless Components Letters, 2010, 20(8): 444-446.

[9] Oh S, Shin B, Jeong J, et al. UWB bandpass filter using dual stepped impedance stub loaded resonators[J]. Microwave \& Optical Technology Letters, 2014, 56(2): 448-450.

[10] Chu Q X, Wu X H, Tian X K. Novel UWB Bandpass Filter Using Stub-Loaded Multiple-Mode Resonator[J]. IEEE Microwave \& Wireless Components Letters, 2011, 21(8): 403-405.

[11] Wei C, Wang X H, Yong T, et al. A UWB bandpass filter with high performances using pair of multiplemode resonators[J]. Microwave \& Optical Technology Letters, 2011, 54(2): 289-292.

[12] Hui C. Compact UWB BPF using coupled-line triple-mode resonator[J]. Electronics Letters, 2013, 49(24): 1541. 
[13] Liang J G, Zhang X, Sun L. Compact UWB bandpass filter with triple notched bands using quadruplemode resonator[C] IEEE International Conference on Microwave \& Millimeter Wave Technology, IEEE, 2016.

[14] Shi X, Xi X, Liu J, and Yang H. Novel ultra-wideband (UWB) bandpass filter using multiple-mode resonator[J]. IEICE Electronics Express, 2016, 13(11): 1-7.

[15] Jhariya D, Azad A R, Mohan A, et al. A compact modified U - shaped UWB bandpass filter[J]. Microwave \& Optical Technology Letters, 2015, 57(9): 2172-2175.

[16] Zhu H, Chu Q X. Compact ultra-wideband (UWB) bandpass filter using dual-stub-loaded resonator (DSLR)[J]. IEEE Microwave \& Wireless Components Letters, 2013, 23(10): 527-529.

[17] Song C, Sha L, Wang Y, et al. A novel ultra-wideband filter with wider stopband employing multiplemode resonator and low-pass filter[C] International Conference on Electronic Packaging Technology, IEEE, 2015.

[18] Aditi S, Somdotta R C and Santanu D. Design of an UWB Bandpass Filter Using Dual MMR with Highly Attenuated Upper Stopband Using DGS[J]. International Journal of Wireless and Microwave Technologies, 2018, 8: 58-69.

[19] Hao Z C, Hong J S. UWB Bandpass Filter Using Cascaded Miniature High-Pass and Low-Pass Filters With Multilayer Liquid Crystal Polymer Technology[J]. IEEE Transactions on Microwave Theory \& Techniques, 2010, 58(4): 941-948.

[20] Wu X H, Chu Q X, Tian X K, et al. Quintuple-Mode UWB Bandpass Filter With Sharp Roll-Off and Super-Wide Upper Stopband[J]. IEEE Microwave \& Wireless Components Letters, 2011, 21(12): 661663.

[21] Xia X, Cheng X, Chen F, et al. Compact UWB bandpass filter with sharp roll-off using APCL structure[J]. Electronics Letters, 2018, 54(13): 837-839.

\section{Authors' Profiles}

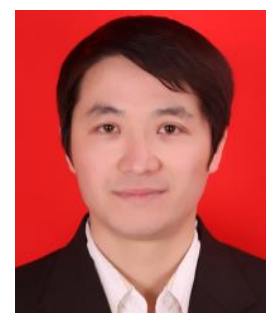

Lei Bai was born in Xianyang, Shaanxi, China. He received his Master's Degree in Signal and Information Processing from Xi'an University of Technology in 2012. He is currently a doctor at school of microelectronics, Xidian University, Xi'an, China. His research interests include passive filter design, high-speed integrated circuits, etc.

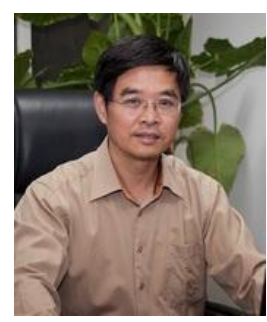

Yiqi Zhuang ( born in 1957 ) received the Ph.D. from Xidian University in 1995. He is the director of the National Integrated Circuit Talents Training Base, professor and doctoral supervisor. His current research interests include communication and power system integration, short-distance wireless communication, microelectronic device noise and reliability application technology, etc. 
Zhibin Zeng, Ph. D., Associate Professor and Master's Tutor, School of Microelectronics, Xidian University. As a technical leader, he has completed the design of wireless Bluetooth system, wireless WIFI system and signal integrity circuit. He has published more than 10 articles on EI and SCI, and applied for more than 10 patents. At present, research has been carried out in the fields of high-speed circuit design, embedded systems and high-speed integrated circuits.

How to cite this paper: Lei Bai, Yi-qi Zhuang, Zhi-bin Zeng, " Compact Ultra-wideband Bandpass Filter with Wide Fractional Bandwidth and High Skirt Selectivity", International Journal of Wireless and Microwave Technologies(IJWMT), Vol.9, No.6, pp. 31-39, 2019.DOI: 10.5815/ijwmt.2019.06.04 